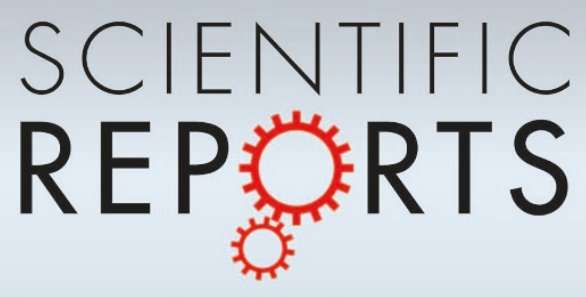

\section{OPEN}

\section{SUBJECT AREAS:}

SOFT MATERIALS

CHARACTERIZATION AND

ANALYTICAL

TECHNIQUES

Received

23 September 2013

Accepted

16 December 2013

Published

15 January 2014

Correspondence and requests for materials should be addressed to S.C. (sahraoui. chaieb@kaust.edu.sa)

\title{
Elasto-plasticity in wrinkled polymerized lipid membranes
}

\author{
Sahraoui Chaieb
}

Division of Physical Science and Engineering, King Abdullah University of Science and Technology (KAUST), Thuwal 23955-6900. KSA.

Biomembranes shown to behave like elastic sheets, can also suffer plastic deformations. Neutron scattering experiments on partially polymerised wrinkled membranes revealed that when a critical degree of polymerisation is crossed, the wrinkled membranes do not resume their spherical shapes. Instead they remain wrinkled and rigid while their non-polymerised counterparts resume their spherical floppy shapes. The yield stress of these membranes, measured for the first time via the fractal dimension, is intimately related to the degree of polymerisation probably through a $2 \mathrm{D}$ disorder that quenches the lateral diffusion of the lipid molecules. This work might shed light on the physical reason behind the irreversible deformation of echinocytes, acanthocytes and malaria infected red blood cells.

ipid membranes mimic red blood cells (RBC) albeit in an oversimplified way. Besides the lipid bilayer, RBC possess a spectrin mesh for extra shear-resistance. Malaria, acanthocytosis and echynocytosis are examples of pathologies in which RBC suffer deformations that are due to defects in the spectrin network, such as dissociation in the case of malaria, which induces a permanent shape change ${ }^{1}$ or a symmetry breaking between the two leaflets of the lipid membrane in the case of acanthocytes ${ }^{2}$. The mechanics of lipid membranes were modeled using an elastic formalism borrowed from elasic sheets ${ }^{3,4}$ but so far no plastic behavior has been observed nor documented. Plasticity is often attributed to dislocation dynamics in metals ${ }^{5}$ and to volume increases in polymers ${ }^{6}$. It is, however, not clear whether plastic deformations are the result of such kinematics and flows or if they cause them. Stress condensation on ridges $^{7}$ and vertices ${ }^{8}$ during the crumpling process of thin sheets is an example of when the onset of elastoplasticity is induced by volume reduction ${ }^{9,10}$. Whereas crumpling of thin sheets has emerged as an archetype of disordered systems, the aforementioned studies focused on elastic behavior, which restricts the richness of these systems. Large deformations, however, induce real-life sheets to behave plastically. Elastic crumpling is characterized by a hierarchical cascade during which large folds break into smaller folds until compaction". At "weak" confinement, the competition between bending and stretching dominates the self-avoidance of the sheet, whereas at stronger confinement, when making a ball of radius $R$, for instance, the sheet's self-avoidance hinders the formation of smaller folds ${ }^{12}$. The interplay between self-avoidance and elasticity manifests itself in the Flory relationship, an important result from polymer physics, which states that the radius, $R$, of the crumpled configuration scales with the size of the system, $L$, such that $R \sim L^{v} \sim L^{2 / d_{F}}$, where $v$ is the Flory exponent and $d_{F}$ is the fractal dimension that measures how the sheet's size affects the mechanical compaction. This result holds for crumpling at a constant force, and the identity $v=2 / d_{F}$ is for maximally compacted twodimensional surfaces and holds for different materials ${ }^{13-16}$. For elastic sheets, $d_{F} \sim 2.5$. For plastically deformed macroscopic sheets this relationship holds but with a different exponent $v$, and $d_{F}<2.5^{17}$.

At the micro- and nanoscales, conformations of crumpled graphite oxide sheets were probed experimentally using light and X-rays scattering and the fractal dimension was found to be that of deformed elastic sheets with $d_{F}$ $\sim 2.5^{18,19}$. Membranes, made of partially polymerized diacetylenic phospholipids, and cooled below the chain melting temperature, $T_{m}$, undergo a thermodynamic wrinkling transition, similar to the wrinkling of an elastic macroscopic sheet, where the vesicles abruptly fold, wrinkle, collapse and expel their payload ${ }^{20,21}$. Atomic Force Microscope (AFM) profilometry on these crumpled lipid membranes revealed that their surfaces were selfsimilar and behaved like elastic sheets up to the degree of polymerization after which the roughness exponent fell into a different universality class also called a glassy phase $\mathrm{e}^{21}$. The mechanics that describe this phase are still lacking however. Furthermore, profilometry only probes the envelope of the surface of the wrinkled membrane and does not access the convoluted three-dimensional (3D) conformation of the membrane nor does it give sufficient information on the local mechanics of the wrinkled membrane. To probe the mechanical behaviour of these membranes we use a scattering technique. Since X-rays uncontrollably polymerise the membranes, we used 
neutron scattering to gain access to the local structure while measuring the fractal dimension of these self-similar structures ${ }^{18}$. The scattering intensity is proportional to the structure factor $S(Q)=$ $\int d^{3} \mathbf{r} \exp (i \mathbf{Q} \cdot \mathbf{r}) g(r)$, where $g(r)$ is the pair correlation function, which is a power law for a fractal object, and $\pm S(q) \sim 1-(Q R)^{2} / 2+$ $\cdots$ for $Q R \ll 1$, and $S(Q) \sim(Q R)^{-d_{F}}$ for $Q R \gg 1^{22-24}$. Small Angle Neutron Scattering at the Argonne National Laboratory (Illinois) enabled us to probe length-scales from 15 to $1500 \AA$ such that $Q R \gg 1$, where $\mathrm{R}$ is the size of the vesicle (on the order of tens of microns) and $1 / Q$ is the observation scale. We expected that if the wrinkled membranes were self-similar and fractal, the scattered intensity would be expressed as a power-law in $Q$. (Data analysis is explained in the section Materials and Methods).

\section{Results}

Figure 1 left panel presents the plot of the scattered neutron intensity versus the wavenumber for lipid tubules dispersed in water. As shown in the figure, when $Q$ is smaller than $0.1 \AA^{-1}$, the data from all samples fall on a line best fitted to the function $I(Q) \sim Q^{-d_{F}}$, where $d_{F}$ is the fractal dimension. It is a constant and approximately equal to 3, within experimental error, which is characterestic of mass fractals ${ }^{25}$. Around $Q \simeq 0.1 \AA^{-1}$, the intensity has a local peak at $Q=$ $0.094 \AA^{-1}$ after which it drops, following a power law of the form $Q^{-4}$ corresponding to the Porod regime for sharp interfaces ${ }^{26}$. This partial peak corresponds to an interlayer distance of $6.68 \mathrm{~nm}$ for tubules in the crystalline phase where the bilayers consist of tilted chain-frozen lipids. This value agrees with previous results using X-rays ${ }^{27}$. When the tubules are heated to $45^{\circ} \mathrm{C}$, they swell into vesicles with a characteristic bilayer thickness given by the peak in the intensity at $\mathrm{Q}=$ $0.088 \AA^{-1}$ as shown in Figure 1 right panel. This peak's position, which does not depend on the degree of polymerization, corresponds to an interlayer distance, $d=7.14 \mathrm{~nm}$ higher than twice the lipid chain length.

We systematically varied the degree of polymerization and captured the scattering spectra from the membranes. Figure 2a shows the intensities from vesicles with low and high degrees of polymerization when the temperature was kept at $15^{\circ} \mathrm{C}$. Unlike the plots in Figure 1-left panel, the slope of the intensity spectrum for $Q<0.1 \AA^{-1}$ shows a clear dependence on the degree of polymerization. The fractal dimension, retrieved from the slopes for different degrees of polymerization and shown in Figure 2b, remains constant at around 2.5 over a wide range of degrees of polymerization. At a critical degree of polymerization $\phi_{c} \sim 30 \%$ it drops reaching the value of 2.05 when $\phi \sim 40 \%$. This behavior of $d_{F}$ is similar to the behavior of the roughness exponent for these membranes ${ }^{21}$. The surfaces for which $d_{f}<3$ are clearly different from rough surface fractals $(3<$ $\left.d_{F}<4\right)$ or mass fractals $\left(d_{F} \simeq 3\right)$, however. One wonders about the mechanism behind the drop in the value of the fractal dimension when the degree of polymerization crosses a critical value.

Step IV in Figure 3 demonstrates our new capability to heat the dispersed wrinkled vesicles beyond $T_{m}$. When crumpled vesicles that were polymerized below $30 \%$ were heated beyond $T_{m}$, they recovered their spherical shapes. Their corresponding intensities are given in the bottom curve in Figure 2c. Crumpled vesicles that are polymerized above $30 \%$ and heated beyond $T_{m}$ had the same scattering intensity as the ones kept at low temperature as shown in Figure $2 \mathrm{~d}$. The absence of the interlayer peak and the presence of a cross-over at $Q \sim 0.1$ is inherent in a typical thickness with defects in the membrane. Similar cross-overs have been observed in the spectrin networks of red blood cells (RBCs) when probed with light and $\mathrm{X}$-ray scatterings ${ }^{28}$. At polymerizations beyond a critical value $\phi_{c} \sim$ $30 \%$, the vesicles that are heated to temperatures around 45 or $50^{\circ} \mathrm{C}$ do not recover their spherical shapes anymore as shown by the scattering intensity shown in Figure 2d, where the acquisition lasted for 12 hours, as well as by phase contrast microscopy. They were indeed locked in a wrinkled configuration via plastic deformation. The representative peaks observed at higher temperatures disappear and the intensity plots are similar with equal slopes equal to 2.25 . The vesicles do not unwrinkle and stay trapped in a plastic state. The plots in (3-a) and (3-d) are offset vertically for clarity.

\section{Discussion}

As mentioned above, lipid membranes are well described using continuum elasticity theory. Previous experiments on membranes and graphene sheets revealed that their behaviour is universal and scale independent - lipid membranes behave just like macroscopic elastic sheets. Given the plethora of numerical studies that use continuum mechanics to describe lipid membranes ${ }^{29}$, it is then justifiable to extend results from numerical studies on macroscopic sheets to microscopic sheets such as lipid membranes. Recent numerical simulations ${ }^{30}$, in which a thin sheet of lateral dimension $L$ was packed and crumpled into a ball of radius $R$ under constant force, showed that if the scaling behavior $R \sim L^{2 / d_{F}}$ holds, the fractal dimension depends weakly on the applied crumpling force. These simulations further showed that when the material was purely elastic, $d_{F}=2.5$ remained constant; when the material was elastoplastic however, the fractal dimension decreases from 2.5 to lower values, approaching 2, with the yield stress, $\sigma_{y}$, also decreasing. Similar trend was found in real crumpled plastic foils that were experimentally folded into balls ${ }^{17}$. We recall that the Flory exponent, $v$, which relates the lateral
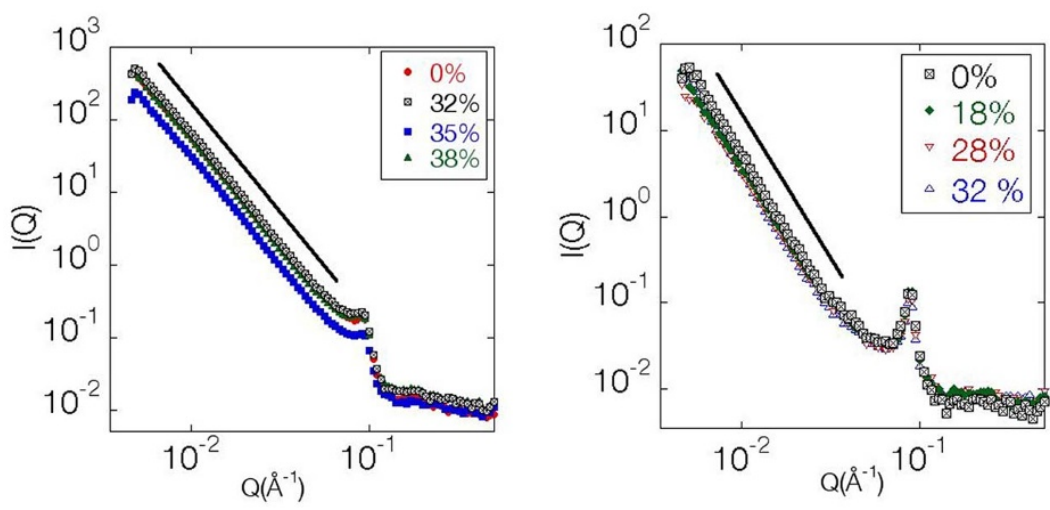

Figure $1 \mid$ Log-Log plots of scattering intensities from a dispersion of vesicles. (a) shows the scattering from a dispersion of thin rods with high aspect ratios forming a network (Step I in Figure 3). The temperature at which this intensity was acquired was $15^{\circ} \mathrm{C}$. All curves lie along a line following $I(Q) \sim Q^{-d_{F}}$, where $d_{F} \simeq 3$. The jump in the slope of the intensity is at $6.68 \mathrm{~nm}$ after which the slope is 4 and in the Porod regime. (b) shows the scattering from a dispersion of vesicles at $45^{\circ} \mathrm{C}$ (Step II in Figure). The characteristic distance, represented by the peak intensity at $Q \simeq 0.088$, corresponds to the bilayer thickness estimated to be $7.14 \mathrm{~nm}$. 


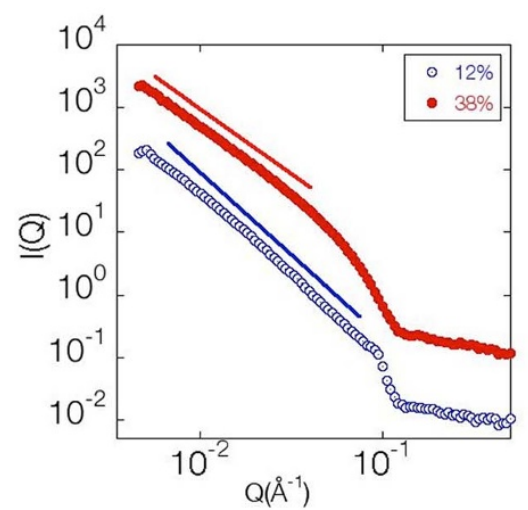

(a)

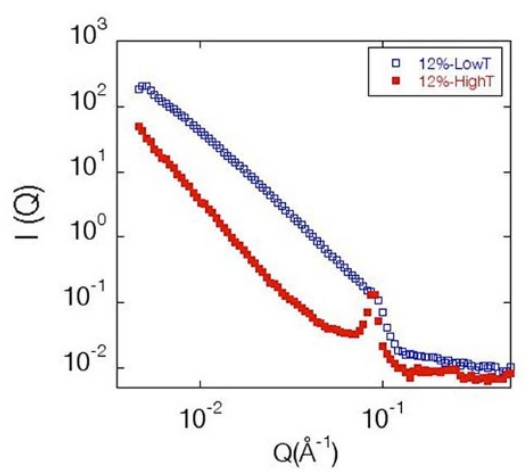

(c)

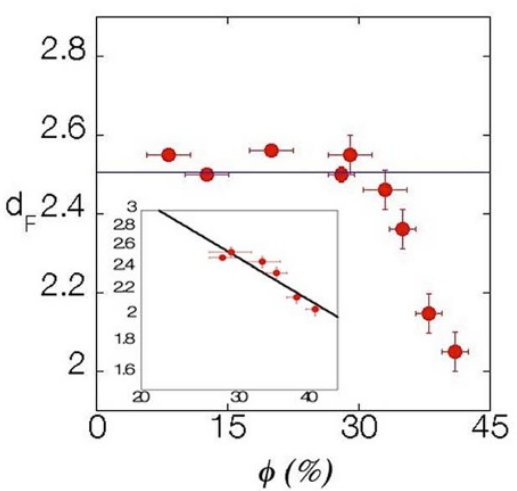

(b)

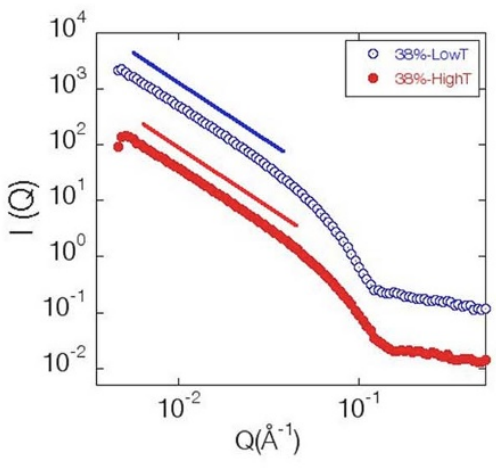

(d)

Figure $2 \mid$ (a)- Log-Log plots of scattering intensities from a dispersion of vesicles at a low degree of polymerization and a high degree of polymerization at $15^{\circ} \mathrm{C}$ (Step III). The lines in a show the power law fit $I(Q) \sim Q^{-d_{F}}$, where the fractal dimension, $d_{F}$ is 2.25 for the upper curve and 2.55 for the lower curve. (b) gives the fractal dimensions of the wrinkled membrane as a function of the degree of polymerization, $\phi$. The error bars at high $\phi$ are due to the variation in the value of $d_{F}$ when the fitting window is varied. The inset shows that $d_{F} \sim \phi^{-0.5}$. (c) shows the intensity at low $\phi$ at low and high-T. The red curve (bottom curve-filled squares) resembles Figure for vesicles. The blue curve (top curve-empty squares) is the intensity at low-T when the vesicles wrinkle. (d) shows the intensity at $\phi>\phi_{c}$ at low and high-T. The blue curve (top curve-empty circles) is the intensity at low temperature. The red curve (bottom curve-filled circles) is the intensity at high-T and it does not look like the curves in Figure 1 nor Figure $2 \mathrm{c}$.

dimension to the size of the confining space or the packed configuration through $R \sim L^{v}$, is related to the fractal dimension, for maximum packing only, via the relation $v=D / d_{F}$, where $D=2$ is the dimension of the sheet ${ }^{14}$. Our observations here allow us to draw various conclusions. Figure $2 \mathrm{~b}$ shows that the membrane has properties similar to an elastic sheet (i.e. mylar or graphene ${ }^{18}$ ) at low degrees of polymerisation and to that of a plastic sheet (i.e. aluminum foil ${ }^{17}$ ) at high degrees of polymerisation. Thus, the two distinct states we observed are related to the transition from elastic to elastoplastic behavior. Moreover, increasing the degrees of polymerization causes the membrane to behave elastoplastically and the yield stress becomes a decreasing function of the degree of polymerization, $\sigma_{y} /$ $E=f(\phi)$, where $E$ is the Young's modulus. Based on the analogies above and that lipid membranes have similar behaviour to macroscopic elastic sheets, we can estimate the value of the yield stress for these polymerised membranes by comparing the values of the fractal dimension for a given yield stress reported $\mathrm{in}^{30}$ to the fractal dimensions reported in this study. $\operatorname{In}^{30}$ the universality of the folding pattern was a function of the ratio of the length of the sheet to its thickness which indicates that the scale of the sheet is irrelevant as long as the ratio of the folds to the bilayer's thickness is above 100 . The non-universality and the dependence of the crumpling on this ratio was attributed to plastic yielding. We find that $\left(\phi ; \sigma_{y} / E\right)=$ $(35 \% ; 0.05),(36.8 \% ; 0.01),(39 \% ; 0.002)$. While the elastoplasticity of macroscopic objects has been characterized in three dimensions only, it is possible to relate elastoplasticity to glassy behavior at the microscopic level in lipid membranes ${ }^{31}$ where defects (disclinations or dislocations) created by the polymerization cause the membrane to behave like a plastic solid ${ }^{32}$. Also, and as shown before and using generalised polarisation measurements ${ }^{21}$, the wrinkling temperature depends on the degree of polymerisation. This is possibly due to a competition between the local intrinsic strain which originates from the polymer network and gives rise to a non-zero gaussian curvature and the possible configurations of the lipids. Although we submitted the vesicles to constant loading $\left[T_{1} \leftrightarrow T_{2}\right.$ ], the wrinkling occured at a temperature that was never below $15^{\circ} \mathrm{C}$. We have compared the fractal dimension values with the values of the roughness exponents and we found a slight correlation beyond the critical polymerization, which we believe is merely coincidental, but surprisingly no correlation below the critical polymerization where both exponents are constant. This could be expected since the roughness exponent measured using AFM underestimated the mechanics of the folding process during the wrinkling which gives rise to an area that is larger than the effective surface measured by the AFM. For rough surfaces generated from fracture for example, the two exponents are linearly dependent ${ }^{33}$.

We now focus on the power-law dependence of the fractal dimension on the degree of polymerisation. According to Figure $2 b$, $I(Q) \sim Q^{-d_{F}}$ and $d_{F} \sim \phi^{-1 / 2}$. The dependence of the fractal dimension on $\phi$ means that the elastic moduli depend on $\phi$. As a first approximation, we assume the Ansatz that $K \sim k_{B} T \phi$, where $K$ is the splay modulus ${ }^{34} . I(Q)$ is proportional to the neutron scattering structure factor, $S(Q)$, which is proportional to the density-density correlation function. If the Hamiltonian used to calculate the 


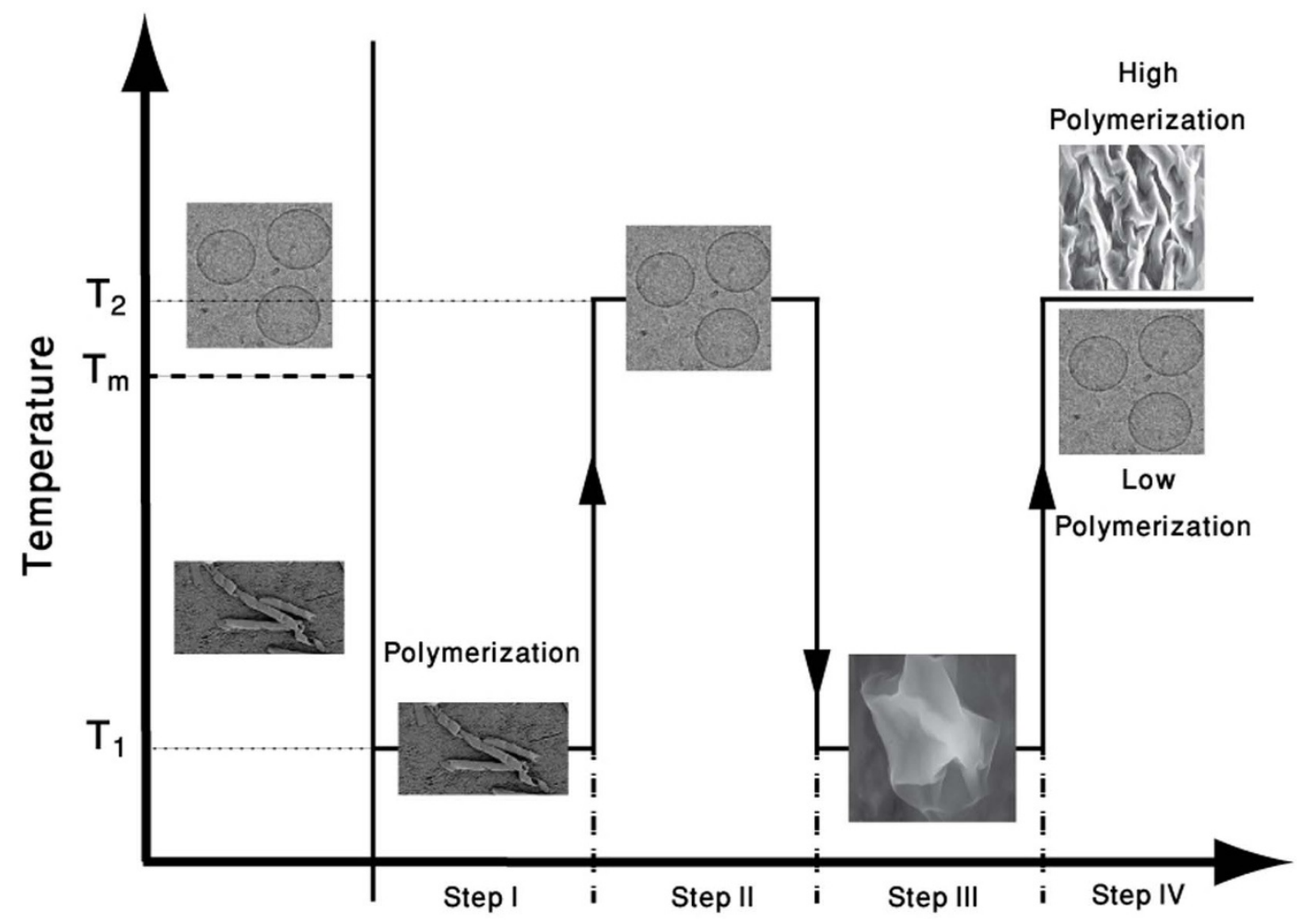

Figure $3 \mid$ Sketch of the of polymerization steps and the wrinkling of the vesicles. Spherical vesicles were initially formed at $T_{2}=45^{\circ} \mathrm{C}$. When they were cooled below $T_{m} \simeq 38.5^{\circ} \mathrm{C}$, they turned into cylindrical tubules. We polymerized them when they were in the tubule phase (Step I) and then, when we heated them, they swelled into spherical vesicles (Step II). When these polymerized spherical vesicles were cooled, they wrinkled when the temperature was $T_{1}$, well below the melting temperature (Step III). When these wrinkled vesicles were then heated back to $T_{2}$ (Step IV), they turned into spheres if the degree of polymerization was less than a critical value or they stayed locked in this configuration if the degree of polymerization was above this critical value. In steps I, II, III and IV, the loadings were identical: $T_{1}=15^{\circ} \mathrm{C}$ and $T_{2}=45^{\circ} \mathrm{C}$.

correlation function is a function only of the Lamé coefficients as in the case of a crystalline membrane or contains only a bending term, the correlation function behaves like a power-law with the exponent depending linearly on the elastic constant ${ }^{35}$. For the exponent to depend on $\phi^{1 / 2}$, the elastic modulus must have the same exponent within the above approximation. As shown earlier, the bilayer becomes compressed when the vesicles are cooled. This suggests that, when calculating the correlation function, we should use the LandauPeierls Hamiltonian that describes the compression of a smectic-A that accompanies splay ${ }^{36}$. This Hamiltonian contains a compression term and is proportional to the compressibility of the layers, $B(\partial u /$ $\partial z)^{2}$, as well as a bending term, $K\left(\partial^{2} u / \partial x^{2}+\partial^{2} u / \partial y^{2}\right)^{2}$. The membranes being polymerized would experience extra stretching on the outer layer like an accordion. Since the membrane is not fully polymerized, we do not need to introduce the Lamé coefficients. If we use this Hamiltonian to calculate the intensity, we find that $I(Q) \sim Q^{-d_{F}}$, where $d_{F}=k_{B} T Q_{m}^{2} / 8 \pi(K B)^{1 / 2}$. Since $K \propto \phi$, then $d_{F} \propto \phi^{-1 / 2}$. It is also possible that the polymer is inducing an unbinding transition of the bilyaer which causes the bilayer to compress when cooled and unbind when heated, due to bending modes in the absence of interlamellar contacts ${ }^{37-39}$.

Noticeably, the topology of the broken spectrin mesh during malaria infection of RBCs is somewhat similar to the random polymerization described above ${ }^{1}$. RBCs experience permanent deformations in the form of echinoytosis either in patients with liver disease ${ }^{40}$ or in patients with Low Density Lipoprotein (LDL) deficiency ${ }^{2}$ and mal$\mathrm{aria}^{41}$. These deformations were interpreted as a consequence of the dissociation of the membrane from the spectrin mesh. When these cells flow into capillaries, they experience large deformations in the form of plastic flow ${ }^{42}$. The random break-up of the spectrin mesh could create defects on the surface of the membranes ${ }^{1}$, which would cause the deformation to become plastic. Such process can be described using the newly developed model of plastic flow in glassy and amorphous materials ${ }^{43,44}$. On the other hand the contour of cancer cells was found to display a fractal behavior that would help identifying cancer cells from normal cells ${ }^{45,46}$.

\section{Methods}

We used polymerizable lipids [1,2-bis(10,12-tricosadiynoyl)-sn-glycero-3-phosphocholine] purchased from Avanti Lipids as powder and stored at $-20^{\circ} \mathrm{C}$. When spherical vesicles made of these lipids are cooled below $T_{m} \simeq 38.5^{\circ} \mathrm{C}$, they undergo a first-order type of shape transformation from spherical vesicles to helical ribbons and tubules ${ }^{27}$. In two-dimensions monolayers of these lipids form spirals below $T_{m}$ and a target pattern above $T_{m}{ }^{21,47}$. The transformation from tubules/ribbons to vesicles when the temperature is increased is also caused by the relaxation of the chiral torque due to thermal fluctuations ${ }^{48}$. We prepared the vesicles using the hydration method: A lipid film from a stock solution of $6 \mathrm{mg} / \mathrm{ml}$ in chloroform was spread on a concave surface of a custom-made teflon container and evaporated over night under a stream of nitrogen. We hydrated the lipid film with deuterated methanol:water (Aldrich, Inc.) $[75: 25(\mathrm{v}: \mathrm{v})]$ to a lipid concentration of $3 \mathrm{mg} / \mathrm{ml}$ and kept it in an oven at $50^{\circ} \mathrm{C}$ for several hours to allow the vesicles to swell. This ensured that the vesicles obtained were Giant Unilamellar Vesicles (GUVs). We heated the vesicles to various temperatures above $T_{m}$ and the results were not affected. We used the hydration method in conjunction with the water/alcohol mixing to produce GUV preferably to the electroformation method because we wanted to avoid any electrostatic effects that migh affect the membranes' properties. Since the lipid molecules were not deuterated, the contrast variation stemmed from the difference between the deuterated solvent and the lipid bilayer. The contrast in the scattering will be between the bilayer and the deuterated water solution. We cooled the vesicles below $\mathrm{T}_{m}$ at a rate of $1^{\circ} \mathrm{C} /$ hour while we kept them in the dark. We polymerized the lipid molecules by exposing the tubules that were kept at $15^{\circ} \mathrm{C}$ to a UV lamp set at $254 \mathrm{~nm}$ and with an intensity of $4 \mu \mathrm{W} / \mathrm{cm}^{2}$. We measured the degree of polymerization as the area under the absorption curve of the polymer using a UV/Vis spectrometer (Genesys 10S $)^{49}$. For our scattering experiments, we prepared various samples each of which corresponded to a separate experiment or step as illustrated in Figure 3. 
The control samples, Step I in Figure 3, included tubules maintained at $15^{\circ} \mathrm{C} \pm$ $0.1^{\circ} \mathrm{C}$ throughout the scattering experiment in 1 - $\mathrm{mm}$-thick quartz cells. We prepared another set of samples by reheating the polymerized vesicles to $45^{\circ} \mathrm{C}$ and keeping them in this state for several hours before letting them cool to $15^{\circ} \mathrm{C}$ when they wrinkled before we started the scattering experiments (Step III). The last set of samples were similar to the second set except that we heated them to $45^{\circ} \mathrm{C}$ and performed the scattering experiments at this temperature. This is shown as Step IV in Figure 3. When changing temperatures, we allowed several hours for the vesicles to equilibrate at the prescribed temperature before we started the data acquisition at the new temperature. The neutron scattering and data acquisition ran for 12 hours continuously.

Data analysis and background correction. The sample's cross section is measured by subtracting the empty cell intensity from the total intensity. The transmission are computed with respect to an empty beam by using a beam stopper and record electronic background that needs to be subtracted. As seen from the intensity at high $\mathrm{Q}(\geq 0.4)$ the incoherent scattering is rather flat and this is due to the solid angle correction from geometric distortion. Although the diminution in the transmission through heavy water is around $3 \%$, which is less than the diminution at high $\mathrm{Q}$ due to geometric distortion, this has been corrected with empty cells containing $\mathrm{D}_{2} \mathrm{O}$. Because we used thin quartz cells that are $500 \mu \mathrm{m}$ thick, multiple scattering was minimized. Substraction of incoherent background which is visible mostly at high $Q$ is performed by empty cell substraction as well as solvent without structure subtraction. At high $Q$ we plot $I(Q) Q^{-4}$ versus $Q$. The slope gives the value of incoherent background which is also reduced by using as little hydrogen atoms as possible. Using a deuterated solvent reduces greatly the incoherent background because of the large differences between the cross section and scattering densities of hydrogen and deuterium atoms. As seen from all scattering plots, the incoherent scattering (at high $Q$ ) is almost flat.

1. Gov, N. \& Safran, S. Red blood cell membrane fluctuations and shape controlled by ATP-Induced cytoskeletal defects. Biophysical Journal 88, 1859-1874 (2005).

2. Lange, Y. \& Steck, T. L. Mechanism of red blood cell acanthocytosis and echinocytosis in vivo. J. Membr. Biol. 77, 153-159 (1984).

3. Canham, P. B. The minimum energy of bending as a possible explanation of the biconcave shape of the human red blood cell. J. Theor. Biol. 26, 61-81 (1970).

4. Helfrich, W. Elastic properties of lipid bilayers: theory and possible experiments. Z Naturforsch C 28, 693-703 (1973).

5. Hill, R. The Mathematical Theory of Plasticity (Oxford University Press, 1998).

6. Robertson, R. E. Theory for the plasticity of glassy polymers. J. Chem. Phys. 44, 3950-3956 (2004).

7. Witten, T. A. Stress focusing in elastic sheets. Rev. Mod. Phys. 79, 643-675 (2007).

8. Cerda, E., Chaieb, S., Melo, F. \& Mahadevan, L. Conical dislocations in crumpling. Nature 401, 46-49 (1999).

9. Chaieb, S. \& Melo, F. Crescent singularities and stress focusing in a buckled thin sheet: mechanics of developable cones. Phys. Rev. E Stat Phys Plasmas Fluids Relat Interdiscip Topics 60, 6091-6103 (1999).

10. Mora, T. \& Boudaoud, A. Thin elastic plates: On the core of developable cones. EPL 59, 41-47 (2002).

11. Sultan, E. \& Boudaoud, A. Statistics of crumpled paper. Phys. Rev. Lett. 96, 136103-136106 (2006)

12. Vliegenthart, G. A. \& Gompper, G. Forced crumpling of self-avoiding elastic sheets. Nat. Mater. 5, 216-221 (2006).

13. Flory, P. J. Principles of Polymer Chemistry (Cornell University Press, 1953).

14. Kantor, Y., Kardar, M. \& Nelson, D. R. Tethered surfaces: Statics and dynamics. Phys. Rev. A 35, 3056-3071 (1987).

15. Gomes, M. a. F. Paper crushes fractally. J. Phys. A: Math. Gen. 20, L283-L284 (1987).

16. Plourabou, F. \& Roux, S. Experimental study of the roughness of crumpled surfaces. Physica A: Statistical Mechanics and its Applications 227, 173-182 (1996).

17. Balankin, A. S., Silva, I. C., Martnez, O. A. \& Huerta, O. S. Scaling properties of randomly folded plastic sheets. Phys. Rev. E 75, 051117-051119 (2007).

18. Wen, X. et al. Crumpled and collapsed conformation in graphite oxide membranes. Nature 355, 426-428 (1992).

19. Spector, M. S., Naranjo, E., Chiruvolu, S. \& Zasadzinski, J. A. Conformations of a tethered membrane: Crumpling in graphitic oxide? Phys. Rev. Lett. 73, 2867-2870 (1994).

20. Mutz, Bensimon \& Brienne. Wrinkling transition in partially polymerized vesicles. Phys. Rev. Lett. 67, 923-926 (1991).

21. Chaieb, S., Natrajan, V. K. \& El-rahman, A. A. Glassy conformations in wrinkled membranes. Phys. Rev. Lett. 96, 078101-078106 (2006).

22. Martin, J. E. \& Hurd, A. J. Scattering from fractals. J. Appl. Cryst. 20, 61-78 (1987).

23. Teixeira, J. Small-angle scattering by fractal systems. J. Appl. Cryst. 21, 781-785 (1988).
24. Bale, H. D. \& Schmidt, P. W. Small-angle x-ray-scattering investigation of submicroscopic porosity with fractal properties. Phys. Rev. Lett. 53, 596-599 (1984).

25. Hammouda, B. A new GuinierPorod model. J. Appl. Cryst. 43, 716-719 (2010).

26. Kratky, O. \& Porod, G. Diffuse small-angle scattering of $\mathrm{x}$-rays in colloid systems. J. Colloid. Sci. 4, 35-70 (1949).

27. Thomas, B. N., Safinya, C. R., Plano, R. J. \& Clark, N. A. Lipid tubule self-assembly: Length dependence on cooling rate through a first-order phase transition. Science 267, 1635-1638 (1995).

28. Schmidt, C. F. et al. Existence of a flat phase in red cell membrane skeletons. Science 259, 952-955 (1993).

29. Abraham, F. F. \& Nelson, D. R. Diffraction from polymerized membranes. Science 249, 393-397 (1990).

30. Tallinen, T., strm, J. A. \& Timonen, J. The effect of plasticity in crumpling of thin sheets. Nat. Mater. 8, 25-29 (2009).

31. Attal, R., Chaieb, S. \& Bensimon, D. Breaking of replica symmetry in a mean-field model of disordered membranes. Phys. Rev. E 48, 2232-2239 (1993).

32. Nelson, D. R. \& Radzihovsky, L. Grain-boundary instabilities and buckling in partially polymerized membranes. Phys. Rev. A 46, 7474-7479 (1992).

33. Bouchaud, E., Lapasset, G. \& Plans, J. Fractal dimension of fractured surfaces: A universal value? EPL 13, 73-79 (1990).

34. Gennes, P.-G. d. Scaling Concepts in Polymer Physics (Cornell University Press, 1979).

35. Sachdev, S. \& Nelson, D. R. Crystalline and fluid order on a random topography. J. Phys. C: Solid State Phys. 17, 5473-5489 (1984).

36. Als-Nielsen, J. et al. Observation of algebraic decay of positional order in a smectic liquid crystal. Phys. Rev. B 22, 312-320 (1980).

37. Lipowsky, R. \& Leibler, S. Unbinding transitions of interacting membranes. Phys. Rev. Lett. 56, 2541-2544 (1986).

38. Wennerstroem, $\mathrm{H}$. The unbinding transition and lamellar phase-lamellar phase coexistence. Langmuir 6, 834-838 (1990).

39. Abraham, F. F. \& Kardar, M. Folding and unbinding transitions in tethered membranes. Science 252, 419-422 (1991).

40. Owen, J. S. et al. Erythrocyte echinocytosis in liver disease. role of abnormal plasma high density lipoproteins. J Clin Invest 76, 2275-2285 (1985).

41. Bessis, M. Living blood cells and their ultrastructure (Springer-Verlag (Berlin and New York) 1973).

42. Li, J., Lykotrafitis, G., Dao, M. \& Suresh, S. Cytoskeletal dynamics of human erythrocyte. PNAS 104, 4937-4942 (2007).

43. Langer, J. S. Shear-transformation-zone theory of plastic deformation near the glass transition. Phys. Rev. E 77, 021502-021515 (2008).

44. Marchenko, V. I. \& Misbah, C. Model of plasticity of amorphous materials. Phys. Rev. E 84, 021502-021508 (2011)

45. Esgiar, A., Naguib, R. N. G., Sharif, B., Bennett, M. \& Murray, A. Fractal analysis in the detection of colonic cancer images. IEEE Transactions on Information Technology in Biomedicine 6, 54-58 (2002).

46. Klein, K., Maier, T., Hirschfeld-Warneken, V. C. \& Spatz, J. P. Marker-free phenotyping of tumor cells by fractal analysis of reflection interference contrast microscopy images. Nano Lett. 13, 5474-5479 (2013).

47. Basnet, P. B., Mandal, P., Malcolm, D. W., Mann, E. K. \& Chaieb, S. Chiral hierarchical self-assembly in langmuir monolayers of diacetylenic lipids. Soft Matter 9, 1437-1446 (2013)

48. Selinger, J. V., MacKintosh, F. C. \& Schnur, J. M. Theory of cylindrical tubules and helical ribbons of chiral lipid membranes. Phys. Rev. E 53, 3804-3818 (1996).

49. Regen, S. L., Czech, B. \& Singh, A. Polymerized vesicles. J. Am. Chem. Soc. 102, 6638-6640 (1980).

\section{Acknowledgments}

We would like to thank Jyotsana Lal for her help, and Elizabeth K. Mann for critical reading of the manuscript. We also thank King Abdullah University of Science and Technology (KAUST) for financial support.

\section{Author contributions}

S.C. performed the analyses discussed the results and wrote the manuscript.

\section{Additional information}

Competing financial interests: The authors declare no competing financial interests.

How to cite this article: Chaieb, S. Elasto-plasticity in wrinkled polymerized lipid membranes. Sci. Rep. 4, 3699; DOI:10.1038/srep03699 (2014).

This work is licensed under a Creative Commons AttributionNonCommercial-ShareAlike 3.0 Unported license. To view a copy of this license, visit http://creativecommons.org/licenses/by-nc-sa/3.0 
SUBJECT AREAS:

SOFT MATERIALS

CHARACTERIZATION AND

ANALYTICAL

TECHNIQUES

\section{SCIENTIFIC REPORTS:}

$4: 3699$

DOI: $10.1038 /$ srep03699

(2014)

Published:

15 January 2014

Updated:

10 December 2014

\section{CORRIGENDUM: Elasto-plasticity in wrinkled polymerized lipid membranes}

\section{Sahraoui Chaieb}

The author neglected to cite a previously published related paper which reports that, when partially polymerized membranes suffer a wrinkling transition, the distribution of wrinkles is lognormal ${ }^{1}$.

In addition, some of the data reported in the manuscript were previously reported in reference 1 . The data in Figure 1 are taken from Figure 3a and Figure 5 in ref. 1; Figure 2b is adapted from Figure 4 in ref. 1.

\section{Reference}

1. Chaieb, S., Málková, Š. \& Lal, J. Why the wrinkling transition in partially polymerized membranes is not universal? Fractal-multifractal hierarchy. Journal of Theoretical Biology 251, 60-67 (2008). 